XLVIth Zakopane School of Physics, International Symposium Breaking Frontiers, Zakopane, Poland, May 16-21, 2011

\title{
Rehydration of CTMA Modified DNA Powders Observed by NMR
}

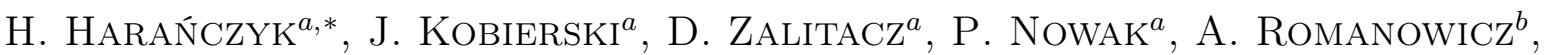 \\ M. MARzeC $^{a}$, J. Niziǫ $^{b}$, E. Hebda $^{c}$ AND J. Pielichowski ${ }^{c}$ \\ ${ }^{a}$ Institute of Physics, Jagiellonian University, Kraków, Poland \\ ${ }^{b}$ AGH University of Science and Technology, Faculty of Physics and Applied Computer Science, Kraków, Poland \\ ${ }^{c}$ Department of Chemistry and Technology of Polymers, Cracow Technical University, Kraków, Poland
}

The rehydration of salmon sperm deoxyribonucleic acid (DNA) and cetyltrimethylammonium chloride $\left(\mathrm{C}_{19} \mathrm{H}_{42} \mathrm{ClN}\right)$ complexes was observed using hydration kinetics, sorption isotherm, and high power proton relaxometry (at $30 \mathrm{MHz}$ ). The hydration kinetics shows (i) a very tightly bound water not removed by incubation over silica gel $\left(A_{0}^{\mathrm{h}}=0.061 \pm 0.004\right)$, (ii) a tightly bound water saturating at $A_{1}^{\mathrm{h}}=0.039 \pm 0.011$, with the hydration time $t_{1}^{\mathrm{h}}=(1.04 \pm 0.21) \mathrm{h}$, a loosely bound water fraction (iii) with the hydration time $t_{2}^{\mathrm{h}}=(19.1 \pm 3.2) \mathrm{h}$ and the contribution progressively increasing with the air humidity. For the hydration at $p / p_{0}=100 \%$, after $t_{0}=(152.6 \pm 2.5) \mathrm{h}$ of incubation the swelling process begins. The swelling time was $t_{3}^{\mathrm{h}}=(12.5 \pm 5.4) \mathrm{h}$, and the swelling amplitude $A_{3}^{\mathrm{h}}=0.140 \pm 0.016$. The sorption isotherm is sigmoidal in form and is fitted by the Dent model with the mass of water saturating primary binding sites $\Delta M / m_{0}=0.102 \pm 0.021$. Proton free induction decay is a superposition of the immobilized proton signal (Gaussian, with $T_{2 S}^{*} \approx 30 \mu \mathrm{s}$ ) and two liquid signal components coming from tightly bound $\left(T_{2 L_{1}}^{*} \approx 100 \mu \mathrm{s}\right)$ and loosely bound water fraction with the amplitude proportional to the mass of water added $\left(T_{2 L_{2}}^{*} \approx 1000 \mu \mathrm{s}\right)$.

PACS: 82.56.Na

\section{Introduction}

While biomaterials often have useful properties, they cannot be easily reproduced. Moreover, biomaterials are a renewable resource, often obtained from post-production wastes. Their nature provides inherent biodegradability. Among all available natural polymers, the DNA is known to have many unusual properties due to its unique double-helix structure [1]. This structure, owing to fundamental biological role [2], has already been thoroughly studied.

A single DNA strand can be viewed as a polymer built of elementary bricks referred to as nucleotides. A nucleotide contains one of four bases (adenine (A), guanine $(\mathrm{G})$, cytosine $(\mathrm{C})$, thymine $(\mathrm{T})$ ), the deoxyribose sugar and the phosphate. All of the bases of a nucleotide can participate in hydrogen bonding. The double helix structure adopted by DNA is composed of two inter-twinned strands. The specific distribution of hydrogen accepting and donating sites favours efficient hydrogen bonds only between adenine and cytosine or between guanine and thymine. As a result, the nucleotides pair with each other in a complementary fashion. In double-stranded DNA, the distance between the two sugarphosphate backbones is the same for each base pair. Consequently, all DNA molecules have the same regular structure in spite of the fact that their nucleotide sequences may be quite different [3].

* corresponding author; e-mail: hubert.haranczyk@uj.edu.pl
The DNA double helix may be imagined as a tunnel of $\pi-\pi^{*}$ stacking nucleic base pairs system available for charge migration. The relatively weak $\pi-\pi^{*}$ electron conjugation renders material with a large optical transparency window (cut off at around $300 \mathrm{~nm}$ due to absorption of bases). It is still discussed whether, regarding the electrical charge transport, DNA belongs to the class of insulators [4], semiconductors [5], conductors [6] or even superconductors [7].

The extracted DNA is soluble exclusively in water. However, due to water high surface tension and low evaporation rate in normal conditions, film processing through standard spin-coating technique does not lead to acceptable results. Moreover, the pristine DNA is very sensitive to hydration [8] which may substantially influence the final device performance [9].

A breakthrough in DNA-based technology was made by demonstrating a series of DNA-cationic surfactant complexes [4]. DNA, which is an anionic polyelectrolyte, can be quantitatively precipitated with cationic surfactant in water by an ion exchange reaction. A smart choice of cationic surfactants pursues to DNA complex insoluble in water and more mechanically and thermally stable than crude DNA. Such complexes were found to be soluble in a range of common alcohols instead of water. Circular dichroism analysis indicated that the right-handed double helix structure of DNA was retained in the bulk.

The best quality films were fabricated from DNA complex with cetyltrimethylammonium (CTMA) (Fig. 1). DNA-CTMA has large band gap of $4.7 \mathrm{eV}$ required for an efficient electron blocking layer (EBL) and thus can 


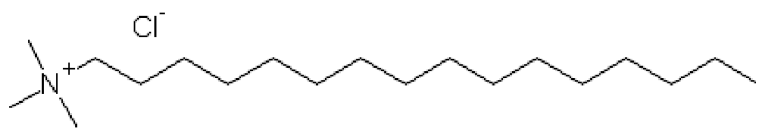

Fig. 1. The cetyltrimethylammonium chloride $\mathrm{C}_{19} \mathrm{H}_{42} \mathrm{ClN}$ (usually abbreviated CTMA).

play a crucial role in improving light emission in OLEDs. Thin films of DNA-CTMA exhibits optical losses as low as $0.2 \mathrm{~dB} \mathrm{~cm}^{-1}$ at $1.550 \mathrm{~nm}$. Relatively large dielectric constant of 7.8 for DNA-CTMA combined with very smooth surface of thin films make it promising material for applications in the FET transistor stuctures.

There were already reported working applications like devices based on second and third order nonlinear optical effects [10], low loss optical waveguides [11], holography [12], organic photovoltaics [13] and organic field effect transistors [14]. DNA may also serve a matrix for other compounds. There have been investigations on lumophore-doped DNA and laser emission from solid state thin films of DNA [15].

Because of these facts, DNA-CTMA is nowadays extensively studied flagship of DNA derived materials applied in photonics and molecular electronics. Nevertheless, some aspects of DNA-CTMA, like kinetics of hydration, are still poorly understood. The effect of mild hydration from gaseous phase is very important as it models the influence of various environmental conditions expired by DNA-CTMA films.

Thus, we studied a number and distribution of water binding sites, sequence and kinetics of their saturation, and the formation of very tightly, tightly, and loosely bound water fractions at rehydration process of DNACTMA complexes, trying to understand the molecular mechanism of structural changes during rehydration from the anhydrous state. We monitored the effect of the sample swelling occurring for hydration from $p / p_{0}=100 \%$.

\section{Materials and methods}

DNA sodium salt, extracted from salmon milt and roe, was purchased from CIST (Chitose Institute of Science and Technology, Japan). It was isolated from frozen salmon milt and roe sacs. The claimed molecular weight was $M_{\mathrm{W}}=10^{6}$ daltons (Da) or 1500 base pairs and purity better than $96 \%$.

A solution of $33.3 \mathrm{ml}$ of deionized water and $200 \mathrm{mg}$ of DNA sodium salt was put in a baker, and stirred mechanically for three hours with a magnet bar at room temperature, which produced clear and homogeneous and viscous solution. Next, the beaker was placed in ultrasounds cleaner for $100 \mathrm{~min}$ at a stabilized temperature to reduce molecular weight of DNA and hence the viscosity. The temperature was close to the ambient to avoid DNA degradation. After such procedure, the solution viscosity decreased. Next, cationic surfactants CTMA were dissolved in deionised water (200 $\mathrm{mg}$ in $150 \mathrm{ml}$ ). Surfactants solutions were added dropwise to the solution of
DNA and constantly stirred for $20 \mathrm{~h}$. The resulted product was filtered off, washed out with deionised water and dried.

Before the hydration courses the air dry samples $\left(\Delta m / m_{0}=0.114 \pm 0.011\right)$ were incubated for $240 \mathrm{~h}$ over silica gel (at relative humidity, $p / p_{0}=0 \%$ ), dehydrating to the hydration level $\Delta m / m_{0}=0.0823 \pm 0.0017$. The dehydration kinetics was well fitted with the single exponential function with the dehydration time constant $t_{1}^{\mathrm{d}}=(4.9 \pm 1.3) \mathrm{h}$.

The hydration time-courses were performed from the gaseous phase with the controlled humidity, at room temperature $\left(t=22^{\circ} \mathrm{C}\right)$, over the surfaces of supersaturated solutions of $\mathrm{KC}_{2} \mathrm{H}_{3} \mathrm{O}_{2}\left(p / p_{0}=23 \%\right), \mathrm{CaCl}_{2}$ $\left(p / p_{0}=32 \%\right), \mathrm{K}_{2} \mathrm{CO}_{3}\left(p / p_{0}=44 \%\right), \mathrm{Na}_{2} \mathrm{Cr}_{2} \mathrm{O}_{7}\left(p / p_{0}=\right.$ $52 \%), \mathrm{NH}_{4} \mathrm{NO}_{3}\left(p / p_{0}=63 \%\right), \mathrm{Na}_{2} \mathrm{~S}_{2} \mathrm{O}_{3}\left(p / p_{0}=76 \%\right)$, $\mathrm{K}_{2} \mathrm{CrO}_{3}\left(p / p_{0}=88 \%\right), \mathrm{Na}_{2} \mathrm{SO}_{4}\left(p / p_{0}=93 \%\right), \mathrm{K}_{2} \mathrm{SO}_{4}$ $\left(p / p_{0}=97 \%\right)$, and over a water surface $\left(p / p_{0}=100 \%\right)$.

After completing the hydration courses, the dry mass of the samples was determined after heating at $70^{\circ} \mathrm{C}$. After heating for $30^{\prime}$, the sample was weighted, then heating was continued. No further decrease in sample mass was recorded for 5 days of drying. The thermal decomposition of DNA-CTMA systems starts at $110^{\circ} \mathrm{C}$, as measured using DSC, although the thermal decomposition of native DNA starts at $200^{\circ} \mathrm{C}[16]$.

Proton free induction decays (FIDs) were recorded on WNS HB-65 high power relaxometer (Waterloo NMR Spectrometers, St. Agatha, Ontario, Canada). The resonance frequency was $30 \mathrm{MHz}$ (at $B_{0}=0.7 \mathrm{~T}$ ); the transmitter power was $400 \mathrm{~W}$; the pulse length $\pi / 2=1.5 \mu$ s. Data were acquired using Compuscope 2000 card in an IBM clone computer, controlling the spectrometer, and averaged over 2000 accumulations. Repetition time was $2 \mathrm{~s}$. The measurements were performed at room temperature $\left(t=22^{\circ} \mathrm{C}\right)$.

The data were analyzed using the one-dimensional, FID analyzing procedure of the two-dimensional (in time domain) NMR signal-analyzing program CracSpin written at the Jagiellonian University, Cracow [17]. The swelling was fitted using program Origin, and the threshold function for the triggering of the swelling process was modeled by the function $\frac{1}{2}\left[1+\tanh \left(\alpha\left(t-t_{0}\right)\right)\right]$ for high values of the parameter $\alpha$.

\section{Results}

\subsection{Hydration kinetics}

The hydration courses for lyophilized DNA-CTMA complexes performed from the gaseous phase (see Fig. 2) are fitted well by single exponential function for $p / p_{0} \leq$ $32 \%$ :

$$
\Delta m(t) / m_{0}=A_{0}^{\mathrm{h}}+A_{1}^{\mathrm{h}}\left[1-\exp \left(-t / t_{1}^{\mathrm{h}}\right)\right],
$$

where $\Delta m / m_{0}$ is the relative mass increase, $A_{0}^{\mathrm{h}}$ is the saturation level for very tightly bound water fraction (i) not removed by incubation over silica gel $\left(p / p_{0}=0 \%\right)$, $A_{1}^{\mathrm{h}}$ is the saturation level for the tightly bound water 
fraction (ii), and $t_{1}^{\mathrm{h}}$ is the corresponding hydration time constant. The averaged over the all target humidities, $A_{0}^{\mathrm{h}}$ equals $0.061 \pm 0.004$. For tightly bound water component $A_{1}^{\mathrm{h}}=0.039 \pm 0.011$, whereas the hydration time $t_{1}^{\mathrm{h}}=(1.04 \pm 0.21) \mathrm{h}$. For the hydration from $p / p_{0}$ between $32 \%$ and $100 \%$ the loosely bound water fraction appears and the hydration course is better fitted by the two-exponential function

$$
\begin{aligned}
& \Delta m(t) / m_{0}=A_{0}^{\mathrm{h}}+A_{1}^{\mathrm{h}}\left[1-\exp \left(-t / t_{1}^{\mathrm{h}}\right)\right] \\
& \quad+A_{2}^{\mathrm{h}}\left[1-\exp \left(-t / t_{2}^{\mathrm{h}}\right)\right],
\end{aligned}
$$

where $A_{2}^{\mathrm{h}}$ is the saturation level for the loosely bound water fraction (iii), and $t_{2}^{\mathrm{h}}=(19.1 \pm 3.2) \mathrm{h}$ is the corresponding hydration time constant. The constant $A_{2}^{\mathrm{h}}$ increases gradually with the relative humidity of the atmosphere. The third exponential in hydration kinetics curve was not observed, as it was in salmon sperm DNA not treated by the use of surfactant [8].

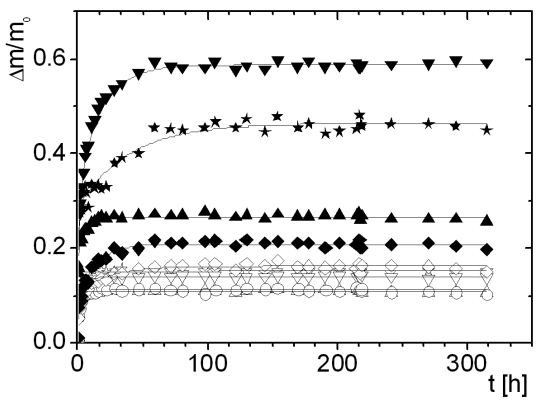

Fig. 2. The hydration kinetics for DNA-CTMA complexes performed from the gaseous phase at different values of relative humidity $p / p_{0}$, recorded as relative mass increase expressed in units of dry mass $\Delta m / m_{0}$. Targets humidity: $p / p_{0}=23 \%$ - open circles, $p / p_{0}=32 \%$ open triangles, $p / p_{0}=44 \%$ - open reverse triangles, $p / p_{0}=52 \%$ - open diamonds, $p / p_{0}=63 \%$ - open starlets, $p / p_{0}=76 \%$ - closed diamonds, $p / p_{0}=88 \%$ - closed triangles, $p / p_{0}=93 \%$ - closed starlets, $p / p_{0}=97 \%$ - closed reversed triangles. The error bars are within the plot symbols.

\subsection{Swelling}

The DNA-CTMA lyophilizate rehydrated from $p / p_{0}=$ $100 \%$ revealed the delayed hydration process starting at $t_{0}=(152.6 \pm 2.5) \mathrm{h}$. Two-step function model, which describes the swelling process, was fitted to the data

$$
\begin{aligned}
& \Delta m(t) / m_{0}=A_{0}^{\mathrm{h}}+A_{1}^{\mathrm{h}}\left[1-\exp \left(-t / t_{1}^{\mathrm{h}}\right)\right] \\
& +A_{2}^{\mathrm{h}}\left[1-\exp \left(-t / t_{2}^{\mathrm{h}}\right)\right] \\
& + \begin{cases}0, & t<t_{0}, \\
A_{3}^{\mathrm{h}}\left[1-\exp \left(-t / t_{3}^{\mathrm{h}}\right)\right], & t>t_{0},\end{cases}
\end{aligned}
$$

where $A_{3}^{\mathrm{h}}=0.140 \pm 0.016$ was the swelling amplitude, and $t_{3}^{\mathrm{h}}=(12.5 \pm 5.4) \mathrm{h}$ was the swelling time. The amplitude of the swelling was much smaller than for salmon sperm DNA and started after significantly shorter delay [8]. This may suggest differences in outer surface of the DNA-CTMA lyophilizate grains (Fig. 3).

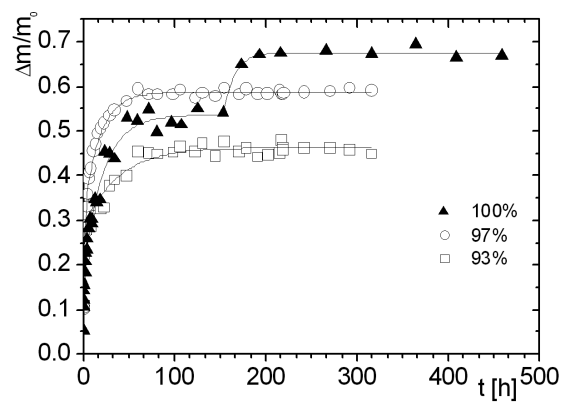

Fig. 3. The swelling process observed at hydration kinetics for DNA-CTMA complexes performed from the gaseous phase. The presented hydration courses were performed at $93 \%$ (open squares), at $97 \%$ (open circles) and at $100 \%$ (closed triangles). Slightly decreased saturation hydration level for the last sample reflected the sample variability.

\subsection{Sorption isotherm}

For DNA-CTMA complexes the total saturation hydration level, $C^{\mathrm{h}}$, was calculated as

$$
C^{\mathrm{h}}=\sum_{i=0}^{n} A_{i},
$$

where $n$ equals 1 or 2 depending on hydration level (see Eq. (1a), (1b)), and subsequently was taken for the construction of sorption isotherm.

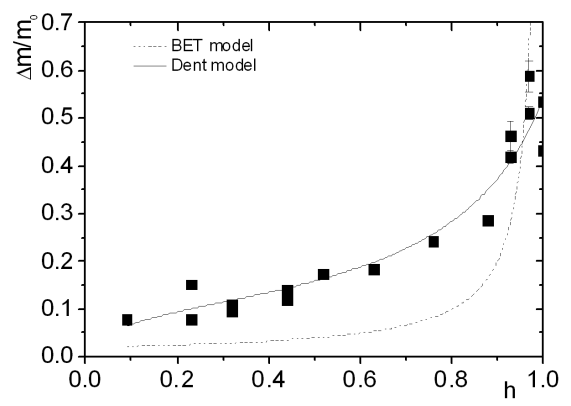

Fig. 4. The sorption isotherm for DNA-CTMA complexes. The values of $h\left(=p / p_{0}\right)$ represent the relative humidity, and the values of relative mass increase, $\Delta m / m_{0}$, are defined as the $C^{\mathrm{h}}$ values taken from Eq. (1a) and (1b). Closed squares - measured points, solid line - the Dent model, dotted line - BET model.

The sorption isotherm is approximately sigmoidal (Fig. 4), which is well fitted by the theoretical sorption models dealing with the two types of water binding sites, (i) "primary" water binding sites (directly to the adsorbent surface); and (ii) "secondary" water binding sites (to the bound water molecules). Either in the BrunauerEmmett-Teller (BET) [18] model or the Dent [19] model 
the sorption isotherm is expressed by

$$
C^{\mathrm{h}}(h)=\frac{\Delta M}{m_{0}} \frac{b_{1} h}{(1-b h)\left(1+b_{1} h-b h\right)},
$$

where $h$ is relative humidity, $p / p_{0}$ is expressed in absolute units, and $\Delta M / m_{0}$ is the mass of water saturating primary binding sites. If $S_{i}$ is the number of binding sites covered by $i$ water molecules, and the contribution of empty primary binding sites is $S_{0}$, the parameter $1 / b_{1}$ is defined as $S_{0} /\left.S_{1}\right|_{h=1}=1 / b_{1}$. Both considered models of sorption isotherm differ in population of subsequent layers of secondary bound water, $S_{n} /\left.S_{n-1}\right|_{h=1}$. BET model takes $b=S_{n} /\left.S_{n-1}\right|_{h=1}=1$, whereas in the Dent model this number may be varied between 0 and 1 .

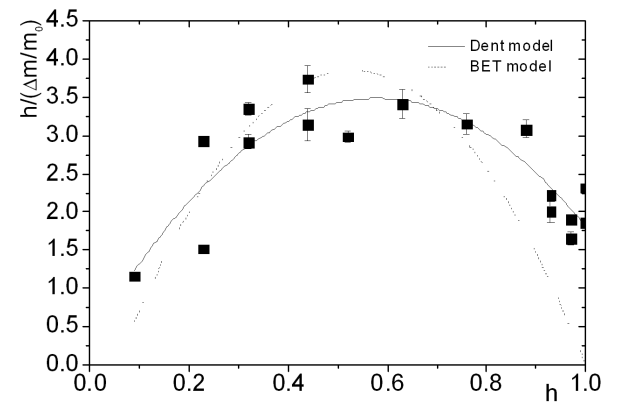

Fig. 5. Parabolic form of the Dent and BET model of the sorption isotherm for DNA-CTMA complexes [closed squares - experimental data, solid line - fitted Dent model (Eq. (3b)), dotted line - fitted BET model (Eq. (3a))].

To test the relevance of the model used, the sorption isotherm is usually presented in parabolic form (see Fig. 5), which for BET model is expressed as

$$
\frac{h}{\Delta m / m_{0}}=A+B h-(A+B) h^{2},
$$

whereas for the Dent model as

$$
\frac{h}{\Delta m / m_{0}}=A+B h-C h^{2},
$$

where parameters $\frac{\Delta M}{m_{0}}, b, b_{1}$ are connected with $A, B, C$ by the formulae

$$
b=\frac{\sqrt{B^{2}+4 A C}-B}{2 A}, \quad b_{1}=\frac{B}{A}+2 b, \quad \frac{\Delta M}{m_{0}}=\frac{1}{A b_{1}} .
$$

For DNA-CTMA complexes the sorption isotherm is much better described by the Dent model $(b=0.814 \pm$ $0.040)$ than by BET model. This value resembles the obtained for lyophilized native $(b=0.896)[20]$ or modified photosynthetic membranes $(b=0.863)$ [21].

The mass of water saturating primary water binding sites was $\Delta M / m_{0}=0.102 \pm 0.021$, which is the value close to that obtained from hydration kinetics for very tightly bound water pool.

For the DNA-CTMA complex, the empty binding sites proportion at $h=1$ equals $1 / b_{1}=6.85 \%$, which is in the range for those measured for native and for modified photosynthetic membranes [20,21], and significantly exceeds the value for solid tissues of living organisms at dehydration, e.g. for Usnea antarctica, $1 / b_{1}=0.008 \%$ [22], for Umbilicaria aprina $1 / b_{1}=0.02$ [23], whereas for Himantormia lugubris $1 / b_{1}=1.11 \%$, and for Caloplaca regalis $1 / b_{1}=1.93 \%[24]$.

\subsection{Proton free induction decays}

The FIDs for protons of the DNA-CTMA complexes, at low hydration levels $\left(\Delta m / m_{0} \leq 0.1\right)$, are well fitted by the superposition of one Gaussian component, with the amplitude $S$, coming from immobilized protons, and one exponential component, $L_{1}$, coming from water tightly bound on the surfaces of the structure (Eq. (7a)):

$$
\mathrm{FID}(t)=S \exp \left(-\left(\frac{t}{T_{2 S}^{*}}\right)^{2}\right)+L_{1} \exp \left(-\frac{t}{T_{2 L_{1}}^{*}}\right)
$$

where $T_{2 S}^{*} \approx 30 \mu$ s is the proton spin-spin relaxation time of solid component taken as the 1/e-value of Gaussian solid signal, and $T_{2 L_{1}}^{*}$ is the relaxation times of proton liquid fraction $L_{1}$. For higher hydration levels a solid Gaussian signal is no longer observed and for $\Delta m / m_{0} \geq 0.2$ a signal from the loosely or free water fraction appears (Eq. (7b))

$$
\mathrm{FID}(t)=L_{1} \exp \left(-\frac{t}{T_{2 L_{1}}^{*}}\right)+L_{2} \exp \left(-\frac{t}{T_{2 L_{2}}^{*}}\right),
$$

where for higher hydration levels loosely bound water component, $L_{2}$, and $T_{2 L_{2}}^{*}$ are the amplitude, and relaxation time of loosely bound water fraction, respectively. A typical FIDs are shown in Fig. 6.

The hydration dependence of the proton relaxation times for fitted components of the FID signal of DNACTMA complexes is presented in Fig. 7.

For the proton fraction $L_{1}$ the value of the decay time does not depend much on the hydration level and equals $T_{2 L_{1}}^{*} \approx 100 \mu \mathrm{s}$. Proton FID time for the $L_{1}$ fraction resembles that for the immobilized (tightly bound) water signal of photosynthetic membranes [20, 21], bark and bast [25], wheat seed [26], lichen thallus [22, 23, 27], dentine and dental enamel [28], and of the controlled pore glasses [29].

For the hydration levels $\Delta m / m_{0} \geq 0.2$ the long, $L_{2}$, signal component appears. The relaxation time $T_{2 L_{2}}^{*} \approx$ $1000 \mu \mathrm{s}$. The $L_{2}$ signal, expressed in units of total liquid signal, $L_{2} /\left(L_{1}+L_{2}\right)$, increases in the intensity with the increasing hydration level (Fig. 8), suggesting that this component comes from water loosely bound or free water in the system. It is an average of some proton subsystems being in fast exchange regime [30]. The spin-spin relaxation times $T_{2 L_{2}}^{*}$ measured in FID experiment are shortened by $B_{0}$ inhomogeneities [31]:

$$
\frac{1}{T_{2}^{*}}=\frac{1}{T_{2}}+\frac{\gamma \Delta B_{0}}{2}
$$

where $T_{2}$ is spin-spin relaxation time, $\gamma$ is gyromagnetic ratio, and $\Delta B_{0}$ is a change of magnetic field $B_{0}$ within 


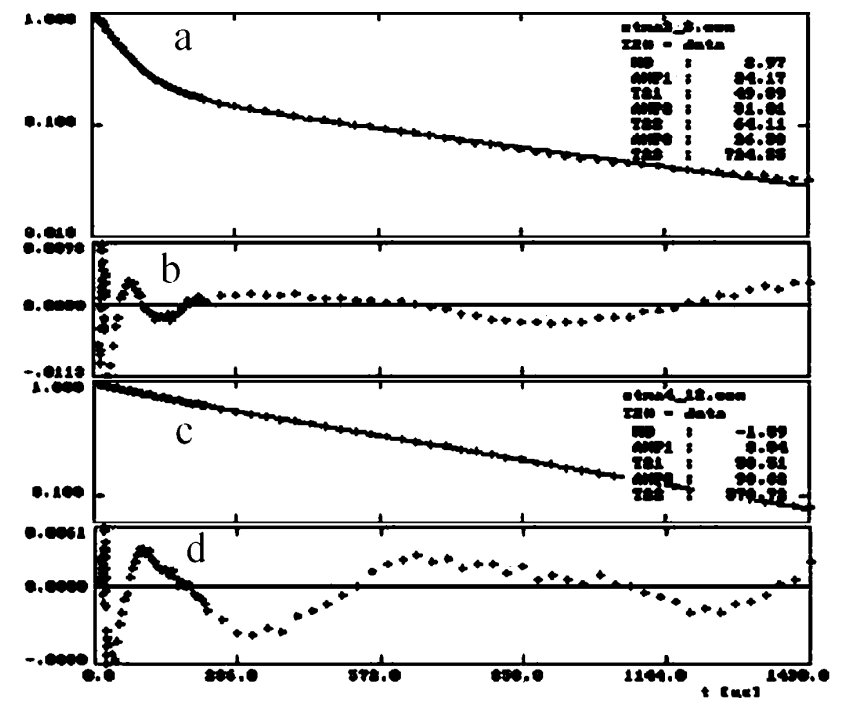

Fig. 6. Proton FID recorded for DNA-CTMA complexes at $30 \mathrm{MHz}$; the pulse length $\pi / 2=1.5 \mu \mathrm{s}$. The relative mass increase was (a) $\Delta m / m_{0}=0.132$, and (c) $\Delta m / m_{0}=0.241 . \quad Y$-axis shows the normalized signal amplitude. The solid line represents a least squares fit of Eq. (7a) and (7b), respectively, to the data ((b) and (d)). The residual function, calculated as the difference between the fitted and the recorded values of the FID signal, does not exceed $1.1 \%$ and $0.8 \%$, respectively.

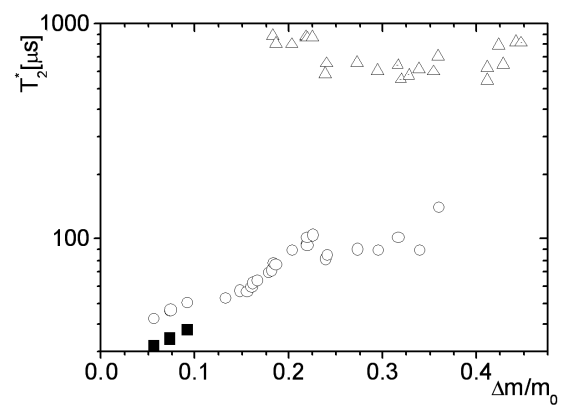

Fig. 7. The hydration dependence of proton FID relaxation times for DNA-CTMA complexes. Solid signal component, $S$ (closed squares), tightly bound water component, $L_{1}$ (open spheres), and loosely bound water, $L_{2}$ (open triangles), component.

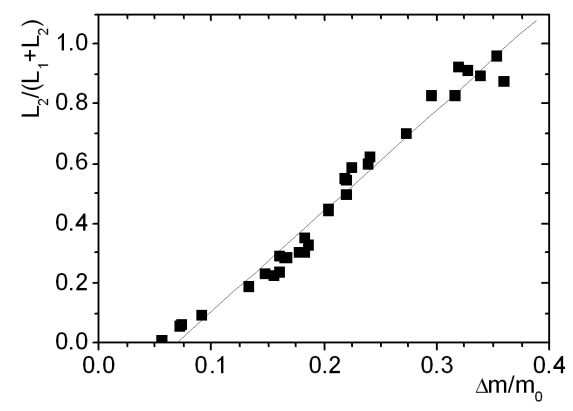

Fig. 8. The $L_{2} /\left(L_{1}+L_{2}\right)$ hydration dependence for rehydrated DNA-CTMA complexes. The solid line was fitted from Eq. (8). the sample; and this is a reason why $T_{2 L_{2}}^{*}$ does not increase for higher hydration levels. Although the solid and short exponential components in the FID experiment are not changed by $\Delta B_{0}$ as compared to those measured by the Carr-Purcell-Meiboom-Gill (CPMG) echo train, the measured $T_{2 L_{2}}^{*}$ are significantly shortened.

The $L_{2}$ signal, expressed in units of total liquid signal, $L_{2} /\left(L_{1}+L_{2}\right)$, almost linearly increases with the increased hydration level and is well fitted by the dependence (Eq. (8)):

$$
\begin{aligned}
& \frac{L_{2}}{L_{1}+L_{2}}\left(\Delta m / m_{0}\right)=(3.39 \pm 0.11) \Delta m / m_{0} \\
& -(0.235 \pm 0.025) .
\end{aligned}
$$

The estimated (Eq. (8)) value of hydration level at which the loosely bound water fraction appears is equal to $\Delta m / m_{0}=0.0693 \pm 0.0077$. This value is slightly lower than that $\left(\Delta M / m_{0}=0.102 \pm 0.021\right)$ obtained as water portion saturating primary water binding sites.

\section{Discussion}

The lengths of fully extended CTMA molecule may be estimated as $l=2.5 \mathrm{~nm}[32]$. However, for the temperatures above main transition $[33,34]$ as the effective lengths of hydrocarbon chain there should be taken $0.75 l$ [35]. This makes $3.75 \mathrm{~nm}$ as an effective diameter of the micelle formed by CTMA in presence of water. This value fits well to the DNA molecule lengths, enabling it to form DNA-CTMA micelle even when a real complex is not built. This might explain very effective dissolution of DNA lyophilizate in presence of CTMA. As solid component of proton FID signal vanished at relatively low hydration level, the detailed analysis of all liquid signal components was more difficult, and it was probably a reason why the estimation of water amount saturating primary water binding sites differs from the saturation value of the immobilized water component, $L_{1}$, from the FID signal.

\section{Acknowledgments}

The research was carried out with the equipment purchased thanks to the financial support of the European Regional Development Fund in the framework of the Polish Innovation Economy Operational Program (contract no. POIG.02.01.00-12-023/08). This project was partially financed by Polish National Science Centre (contract no. 0891/B/H03/2011/40).

\section{References}

[1] A.J. Steckl, Nat. Photon. 1, 3 (2007).

[2] V.A. Bloomfield, D.M. Crothers, I. Tinoco, J.E. Hearst, D.E. Wemmer, P.A. Killman, D.H. Turner, Nucleic Acids: Structures, Properties, and Functions, University Science Books, Sausalito 2000. 
[3] R.H. Horton, L.A. Moran, K.G. Scrimgeour, M.D. Perry, J.D. Rawn, Principles of Biochemistry, Pearson Prentice Hall, New Jersey 2006.

[4] L.L. Wang, J. Yoshida, N. Ogata, Chem. Mater. 13, 1273 (2001)

[5] Y.G. Yang, P.G. Yin, X.Q. Li, Y. Yan, Appl. Phys. Lett. 86, 203901 (2005).

[6] H.W. Fink, C. Schonenberger, Nature 398, 407 (1999).

[7] A.Y. Kasumov, M. Kociak, S. Gueron, B. Reulet, V.T. Volkov, D.V. Klinov, H. Bouchiat, Science 291, 280 (2001).

[8] H. Harańczyk, J. Czak, P. Nowak, J. Nizioł, Acta Phys. Pol. A 112, 397 (2010).

[9] H. Dong Han, N. Hyunsoo, Y. Kyung-Hwa, S. Hye-mi, L. Hae-Yeon, T. Kawai, Chem. Phys. Lett. 355, 405 (2002).

[10] O. Krupka, A. El-Ghayoury, I. Rau, B. Sahraoui, J.G. Grote, F. Kajzar, Thin Solid Films 516, 8932 (2008).

[11] J.G. Grote, J.A. Hagen, J.S. Zetts, R.L. Nelson, D.E. Diggs, M.O. Stone, P.P. Yaney, E. Heckman, C. Zhang, W.H. Steier, A.K.Y. Jen, L.R. Dalton, N. Ogata, M.J. Curley, S.J. Clarson, F.K. Hopkins, J. Phys. Chem. B 108, 8584 (2004).

[12] H.W. Fink, H. Schmid, E. Ermantraut, T. Schulz, J. Opt. Soc. Am. A 14, 2168 (1997).

[13] P. Dutta, S.K. Mandal, J. Phys. D, Appl. Phys. 37, 2908 (2004)

[14] C. Yumusak, T.B. Singh, N.S. Sariciftci, J.G. Grote, Appl. Phys. Lett. 95, 263304 (2009).

[15] Y. Kawabe, L. Wang, S. Horinouchi, N. Ogata, Adv. Mater. 12, 1281 (2000)

[16] S.L. Lee, P.G. Debenedetti, J.R. Errington, B.A. Pethica, D.J. Moore, J. Phys. Chem. B 108, 3098 (2004).

[17] W. Węglarz, H. Harańczyk, J. Phys. D, Appl. Phys. 33, 1909 (2000).
[18] S. Brunauer, P.H. Emmett, E. Teller, J. Am. Chem. Soc. 60, 309 (1938).

[19] R.W. Dent, Textile Res. J. 47, 145 (1977).

[20] H. Harańczyk, A. Leja, K. Strzałka, Acta Phys. Pol. A 109, 389 (2006).

[21] H. Harańczyk, A. Leja, M. Jemioła-Rzemińska K. Strzałka, Acta Phys. Pol. A 115, 526 (2009).

[22] H. Harańczyk, A. Pietrzyk, A. Leja, M. Olech, Acta Phys. Pol. A 109, 411 (2006).

[23] H. Harańczyk, M. Bacior, M. Olech, Antarctic Sci. 20, 527 (2008)

[24] H. Harańczyk, On Water in Extremely Dry Biological Systems, WUJ, Kraków 2003.

[25] H. Harańczyk, W.P. Węglarz, S. Sojka, Holzforschung 53, 299 (1999).

[26] H. Harańczyk, K. Strzałka, G. Jasiński, K. Mosna-Bojarska, Coll. Surf. A 115, 47 (1996).

[27] H. Harańczyk, M. Bacior, P. Jastrzębska, M.A. Olech, Acta Phys. Pol. A 115, 516 (2009).

[28] N. Funduk, G. Lahajnar, L. Miljković, S. Skočajić, D.W. Kydon, L.J. Schreiner, M.M. Pintar, Zobozdrav. Vestn. 41 (Suppl. 1), 139 (1986).

[29] H. Harańczyk, K.G. Soga, R.J. Rumm, M.M. Pintar Magn. Res. Imag. 9, 723 (1991).

[30] J.R. Zimmerman, W.E. Brittin, J. Phys. Chem. 61, 1328 (1957).

[31] A. Timur, J. Petrol. Technol. 21, 775 (1969).

[32] S.Y. Lee, W.J. Cho, P.S. Hahn, M. Lee, Y.B. Lee, K.J. Kim, Appl. Clay Sci. 30, 174 (2005).

[33] A. Wishnia, J. Phys. Chem. 67, 2079 (1963).

[34] C. McAufliffe, J. Phys. Chem. 70, 1267 (1966).

[35] Ch. Tanford, The Hydrophobic Effect: Formation of Micelles and Biological Membranes, Wiley, New York 1980. 